\title{
Ethical, Social and Legal Consequences of Genomic Medicine and Personalized Psychiatry
}

\author{
Russell D'Souza \\ ${ }^{1}$ Director of Education, UNESCO Chair in Bioethics, Haifa. \\ Corresponding Author: Russell D'Souza \\ Email: russell.f.dsouza@gmail.com
}

\begin{abstract}
Genomics has contributed greatly to our understanding of the molecular basis of disease and, to a lesser but growing extent, to the development of effective interventions. Clinicians and society at large, however, are concerned about the effect genetic knowledge will have on the well-being of individual persons and groups. Much effort is being devoted to trying to anticipate, understand, and address the ethical, legal, social, and political implications of genetics and genomics.

Understanding the social effects of genomics requires an analysis of the ways in which genetic information and a genetic approach to disease affect people individually, within their families and communities, and in their social and working lives. Genomics presents challenges with respect to clinicians' ethical and professional responsibilities, including the appropriate use of genomic information in the health care setting.

Genes affect virtually all human characteristics and diseases. These influences can be ascertained in individual patients through a review of the family history, physical examination, and the use of medical diagnostics. Given the variety of these effects and the limits of knowledge, the term "genetic information" is used in various ways at different times. These various meanings may make sense in context, but confusion can occur requiring the speaker and listener are defining the term in the same way.
\end{abstract}

\section{What people in general are worried about}

The most commonly expressed fear is that genetic information will be used in ways that could harm people - for example, to deny them access to health insurance, employment, education and even loans. Part of that concern is fueled by the growing recognition that health information is not entirely private, despite people's expectations and desires to the contrary. This debate is informed appropriately by the recognition that limiting access to the medical record to the patient and the treating clinician is neither possible nor desirable.

People tend to see genetic information as more definitive and predictive than other types of data, human characteristics are the product of complex interactions over time between genes - both a person's own and those of other organisms - and the environment. It is felt, that the way to allay people's fears about genetics is simply to give them a more realistic understanding of the informative power of these tools. An approach to addressing the social implications of genetics requires knowledge, of how genes are perceived and what is known about their function.

\section{The Issue with Discrimination}

The question of whether genetic information should ever be used to affect one's access to health and other forms of insurance has been a dominant issue of public concern in the past decade. People cite fear of losing insurance as a major reason to avoid genetic testing. Others argue that discrimination by insurance companies is not a problem, often pointing out that few of these cases, which are difficult for employees to win, have been filed. Insurers assert that they do not perform tests to obtain genetic information but argue that they should be free to use such 
information if it is available, citing the need to avoid "moral hazard" - the risk that people who know they will become ill or die soon will try to obtain insurance at regular rates.

The complexity of the issues surrounding discrimination can be illustrated more generally by examining a case involving Burlington Northern Santa Fe Railroad (BNSF). Allegedly relying on the advice of its company physician, who in turn had apparently relied on the representations of a diagnostic company, BNSF began obtaining blood for DNA testing from employees who were seeking disability compensation because of carpal tunnel syndrome that occurred on the job. The employees were reportedly not told the purpose of the tests, which was to detect a mutation associated with hereditary neuropathy with liability to pressure palsies. The company's motive for pursuing testing was never made clear, but it seems reasonable to suspect that BNSF would have tried to deny disability benefits to any employee who had such a mutation, arguing that the mutation, and not the job, caused the carpal tunnel syndrome, the company settled claims brought by its employees for an undisclosed amount of money.

The first step in developing an appropriate response is to determine how the use of genetic information fits within the broader framework of antidiscrimination laws, which were passed to create a certain kind of society, one in which people must be included regardless of race, sex, or disability, even at some cost to employers.

\section{The Challenge of Genomic Medicine with respect to the Physician - Patient Relationship}

Consider the case of a man who died of colon cancer in the 1960s. When the same disease developed in his daughter approximately 25 years later, she obtained her father's pathology slides, discovered that he had had diffuse adenomatous polyposis coli, and sued the estate of her father's surgeon, alleging that the physician should have warned her about her 50 percent risk of having the disorder. An intermediate appellate court in New Jersey ruled that the physician had a duty to warn the daughter directly (she would have been a child at the time of her father's death), perhaps even over her father's objections.

This is only one court's view in one case, but given how much attention it received, it is important to ask whether this was a good result. Two central tenets of Western medicine are that physicians should focus on the interests of their patients and that they should protect the confidentiality of their patients' medical information. Yet the tools of genomic medicine often reveal information about health risks faced not only by patients but also by their relatives. What should clinicians do? It seems clear that they should tell their patients about the risks faced by family members. The harder questions are whether physicians are ethically permitted to contact the relatives themselves, in contravention of traditional patient-centered norms, and whether they should be legally required to do so.

This issue must be viewed in the light of the fact that the duty to protect confidentiality is not absolute. Physicians are required to report numerous infectious diseases and they have been held liable for failing to warn people whom their patients have specifically threatened with violence. The question then becomes more complex: are genetic risks sufficiently similar to these existing exceptions to the requirement of confidentiality that they warrant an exception as well? Over the years, numerous prominent advisory bodies have said no, opining that physicians should be permitted to breach confidentiality to warn third parties of genetic risks only as a last resort to avert serious harm.

\section{Genomic Medicine and Public Health}

Complex questions arises when the government requires testing and interventions. State mandated screening of newborns for metabolic and genetic disorders. Governments undertake many activities to promote health - universal screening of newborns for phenylketonuria, for example, is generally considered a resounding success - but it is worth asking in each case whether there is sufficient justification to pursue mandatory as opposed to voluntary action or to place such activities in the public rather than the private sector. Requiring public health agencies to assume such responsibilities has advantages, such as more transparent accountability to the public and greater uniformity in access and results. Relying on public health entities in matters that directly affect the health of individual persons, however, entails certain risks as well. 
Physicians and patients count on receiving accurate and informative results regardless of whether a private or a public entity is doing the testing. A public health analysis of genomics, of course, involves more than state-run testing. The broadest question is whether the public's health is improved by the knowledge derived. A major determinant is access to testing and to the medical interventions that may be warranted as a result. In our current multiplayer system of health care, people will have widely differing levels of access to these forms of technology. One cannot assume that everyone will reap the benefits of this knowledge.

From a public health perspective, it might do to go one step further and ask whether people will use the test results to alter their behavior in ways that improve health. Some people whom testing identifies as predisposed to cancer subsequently decline to undergo surveillance or other interventions for psychological reasons or because of other demands on their time. Some preventive or therapeutic measures are more likely to be pursued than others; most people find it difficult to take medications for a lifetime or to maintain major lifestyle changes, no matter how important such approaches are for their health.

Public health agencies exist not only to identify barriers to health but also to improve health and health care. Efforts to determine when genetic tests are reliable enough for routine clinical use are quintessential public health activities. The development of strategies to educate health care providers and patients about genomic medicine and to decrease obstacles to health-promoting behavior also falls comfortably within this

\section{Personalized Psychiatry}

Personalized medicine in psychiatry, e.g., in the form of tailored antidepressant or antipsychotic treatment, has already made important progress, notably in terms of adjusted therapeutic doses, and predictable drug responses or drug-induced side effects. Although promising, these opportunities also give rise to numerous scientific, ethical, legal, and social challenges. An adequate assessment of personalized medicine in psychiatry must within all these perspectives be based both on analyses of the science behind pharmacogenomics research to get a realistic view of what can be achieved, and on analyses of the relevant sociopolitical structures surrounding this research.

The ethical considerations that have been considered in terms of adequacy, cost, and therapeutic equity raise no objections to the development of personalized medicine per se in this domain. Rather, they point to the necessity of developing a social infrastructure with adequate guidelines to ensure the responsible implementation of these promising new techniques.

\section{Conclusion}

This brief discussion illustrates public expectations and fears about the effect of genomics, challenges to the goals of antidiscrimination laws and to the nature of the physician-patient relationship, the contrasting perspectives, legal rules that apply to personal medical care, public health and personalized medicine. Acknowledgment and examination of these complex issues are critical for identifying the appropriate ethical principles that should be applied and for creating the necessary legislative and regulatory responses.

\section{REFERENCES AND RECOMMENDED READING}

1. The Health Privacy Project. The state of health privacy: an uneven terrain. (A comprehensive survey of state health privacy statutes). (Accessed July 15, 2003, at http://www.georgetown.edu/research/ihcrp/privacy/statereport.pdf.)

2. Gostin LO. National health information privacy: regulations under the Health Insurance Portability and Accountability Act. JAMA 2001;285:3015-21.

3. Lapham EV, Kozma C, Weiss JO. Genetic discrimination: perspectives of consumers. Science 1996;274:621-4.

4. Hall MA, Rich SS. Patients' fear of genetic discrimination by health insurers: the impact of legal protections. Genet Med 2000;2:214-21.

5. Pokorski RJ. Insurance underwriting in the genetic era. Am J Hum Genet 1997;60:205-16. 
6. Calvo C, Johnson A, eds. Genetics policy report: insurance issues. Washington, D.C.: National Conference of State Legislatures, September 2001.

7. Genetic information and health insurance:enacted legislation as of April 29, 2002. (Accessed July 15, 2003, at http://www.nhgri.nih.gov/Policy_and_public_affairs/Legislation/insure.htm.)



